\title{
Une nouvelle espèce rare d'Euglossini pour la Guyane Française : Eufriesea formosa MOCSARY, 1908. Description de l'allotype mâle (Hymenoptera, Apidae)
}

\author{
Par Gérard LE GofF *
}

\begin{abstract}
I report on the presence in French Guiana of Eufriesea formosa MOCSARY, of which several females and a yet unknown male have been discovered. I also provide an illustrated description of the male allotype of this species.
\end{abstract}

Résumé. Je rapporte ici la présence d'Eufriesea formosa MOCSARY en Guyane Française où plusieurs femelles et le mâle encore inconnu ont été découverts. Je fournis également une description illustrée de l'allotype mâle de cette espèce.

Mots-clés. Apidae, Euglossini, Eufriesea, E. formosa Mocs, allotype mâle.

\section{Introduction}

Des collectes sont opérées depuis 2008 par les collègues de la Société Entomologique des Antilles et de Guyane (SEAG) sur différents sites du Département afin de dresser un large inventaire entomologique. Un très riche lieu de capture est le lieu-dit de "La Montagne des Chevaux" sur la commune de Roura. Les spécimens d'Apidae venant de ce site m'ont été cédés contre leur détermination (essentiellement Xylocopini, Centridini, Euglossini et Ericrocidini).

Dans les exemplaires d'Euglossini d'un premier lot, j'ai d'abord trouvé une nouvelle femelle d'Eufriesea formosa MOCSARY, connue alors par seulement 4 exemplaires (1 de Colombie, 2 du Surinam et 1 du Brésil) (Figure 1). Cette Eufriesea appartient au groupe «pulcra » qui comprend les espèces suivantes : E. pulcra SMITH, 1854 , E. lucifera KIMSEY, 1977, purpurata MOCSARY, 1896, E. formosa MOCSARY, 1908, E. aeniventris MOCSARY, 1865 et E. theresiae MOCSARY, 1908 (Kimsey, 1982). Eufriesea formosa est donc une espèce rare. Elle élabore probablement ses nids dans des termitières construites en hauteur sur les arbres, voire même jusqu'au niveau de la canopée (comme cela est indiqué pour les exemplaires du Surinam - Kimsey, 1982 ; Marcio L. de Oliveira, comm. pers.). Ce mode de nidification semble typique de ce groupe.

Dans un autre lot de spécimens reçus en 2010, j'ai trouvé une nouvelle femelle et surtout, j'ai eu la très agréable surprise de découvrir 3 mâles de l'espèce (capturés en novembre 2009). Cela me permet de décrire l'allotype mâle d'Eufriesea formosa qui jusqu'à présent demeurait inconnu.

* Rue Albert Malet 44, F-76360 Barentin, France. E-mail: legoff.gerard@free.fr

\section{Matériel d'Eufriesea formosa}

2f. - 06.XII.2008 ; 25.I.2010 - Roura - Montagne des Chevaux - EXO N 1758 et No2334 (G. Le Goff leg.). 3m. - .../XI/2009 - même site - Allotype : EXO : N²296 ; paratypes : EXO N²297 - EXO N²298 (G. Le Goff leg.).

Les paratypes mâles seront offerts, l'un à Marcio L. de Oliveira de l'INP de Manaus (Brésil) et l'autre à David Roubik du SITR (République du Panama).

\section{Description de l'allotype mâle}

L'habitus, comme pour les autres mâles du groupe, est très semblable à celui de la femelle (Figure 1). La longueur du corps est de 18-19 mm ; la langue mesure $9 \mathrm{~mm}$ et atteint les coxae postérieures (comme chez la femelle).

Tête : Vert jaunâtre avec des soies jaunes. Le clypeus présente un aplat central ovalaire avec une ligne médiane irrégulière, en léger relief, imponctuée dans ses 2/3 apicaux et s'effaçant dans une forte ponctuation vers sa base. Plus importante dépression vers les fosses tentoriales antérieures. Labrum avec deux crêtes longitudinales arrondies à leur extrémité et un renflement triangulaire médian à sa base (Figure 2A).

Thorax : Tégument bleu avec une pilosité noire. L'aile antérieure mesure 12,5 $\mathrm{mm}$. Patte antérieure à brosse tarsale dorée. Patte médiane avec la tache veloutée antérieure s'effilant depuis la base sur la moitié de la longueur du fémur jusqu'à la petite brosse médiotibiale (Figure 2B) ; peigne basitarsal présent sur le lobe postérieur arrondi à reflets bleus.

Pattes postérieures: sur la face dorsale, le fémur se termine par 2 pointes latérales; la pointe interne est un peu plus longue et écourtée à son extrémité. Entre elles deux, la zone avec la fente de l'organe poreux (stockage des 


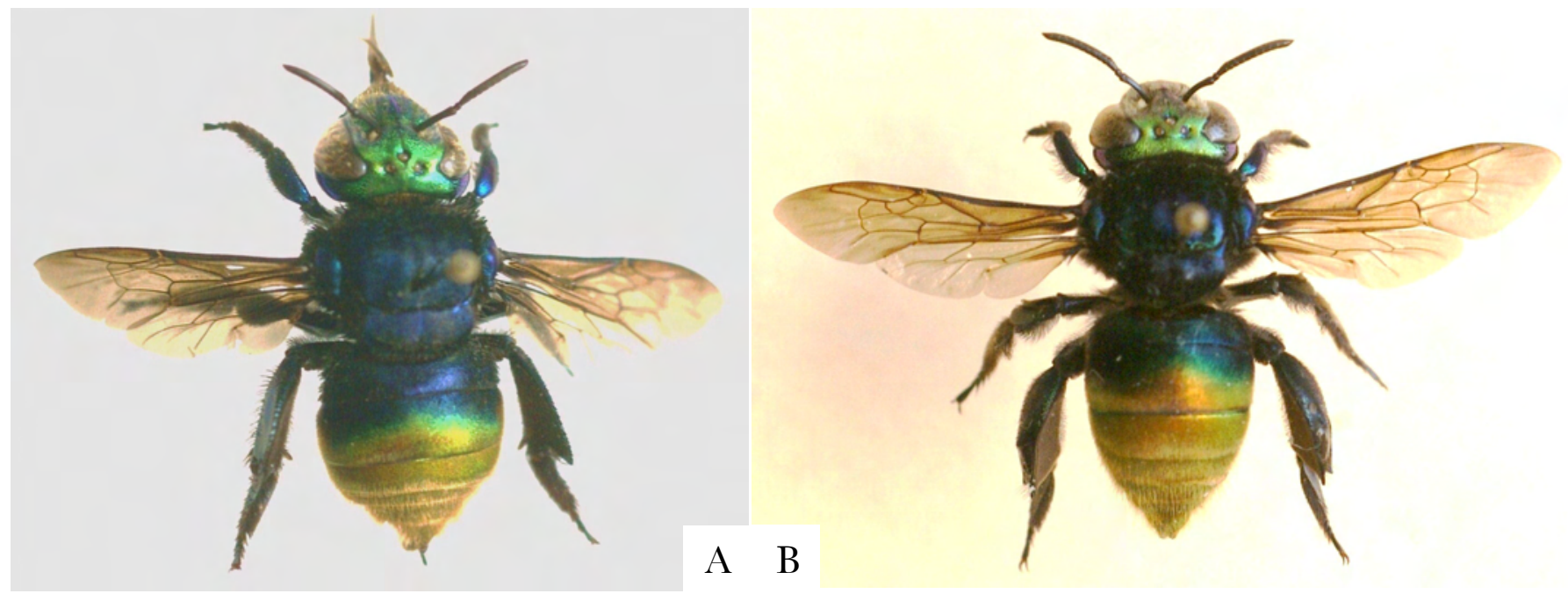

Figure 1. Habitus d'Eufriesea formosa Mocsary femelle (Hym. Apidae) (A) et de l'allotype mâle (B) (Photos G Le Goff)

fragrances) s'étend sur les 2/5 de la longueur du fémur. Sur la face interne, on a une pointe au-dessus de chacun des 2 éperons (Figures 2C \& 2D).

Abdomen : T1 noir bleuté avec des soies noires et jaunes. T2 avec la partie antérieure de la même couleur que $\mathrm{T} 1$, avec des soies noires ; la partie postérieure est verte, allant d'un vert bleuté à un vert jaunâtre vers l'apex. Les tergites suivants sont vert jaunâtre avec de longues soies jaunes.

Genitalia : le lobe dorsal du gonostylus est un peu plus court que son lobe ventral. Le lobe dorsal de la gonocoxa est un peu plus long que la moitié du gonostylus (Figures 2E \& 2F). Sternite 8 : ce sternite présente deux pointes apicales en vue latérale (Figures $2 \mathrm{G} \& 2 \mathrm{H})$.
Sternite 7 : les lobes sont deux fois plus longs que larges à la base. ; la distance entre les lobes à leur sommet est égale à la largeur d'un lobe à sa base (Figure 2I). Ces détails et proportions sont utilisés par L.S. Kimsey dans sa monographie de 1982, pour distinguer les mâles des différentes espèces.

\section{Remerciements}

Je remercie l'équipe de la SEAG qui m'a confié une partie de leur matériel, plus particulièrement Marcio L. de Oliveira et David Roubik pour leur intérêt et leurs échanges de courriers électroniques. Un grand merci aussi à mon ami Nicolas Vereecken.

\section{Références bibliographiques}

Kimsey LS, 1982. Systematics of Bees of the Genus Eufriesea (Hymenoptera, Apidae). University of California Press. Berkeley, Los Angeles and London.

Mocsary A, 1908. Species tres novae magnificae generis Euglossa. Annales historico-naturales Musei nationalis hungarici 6: 580-583.
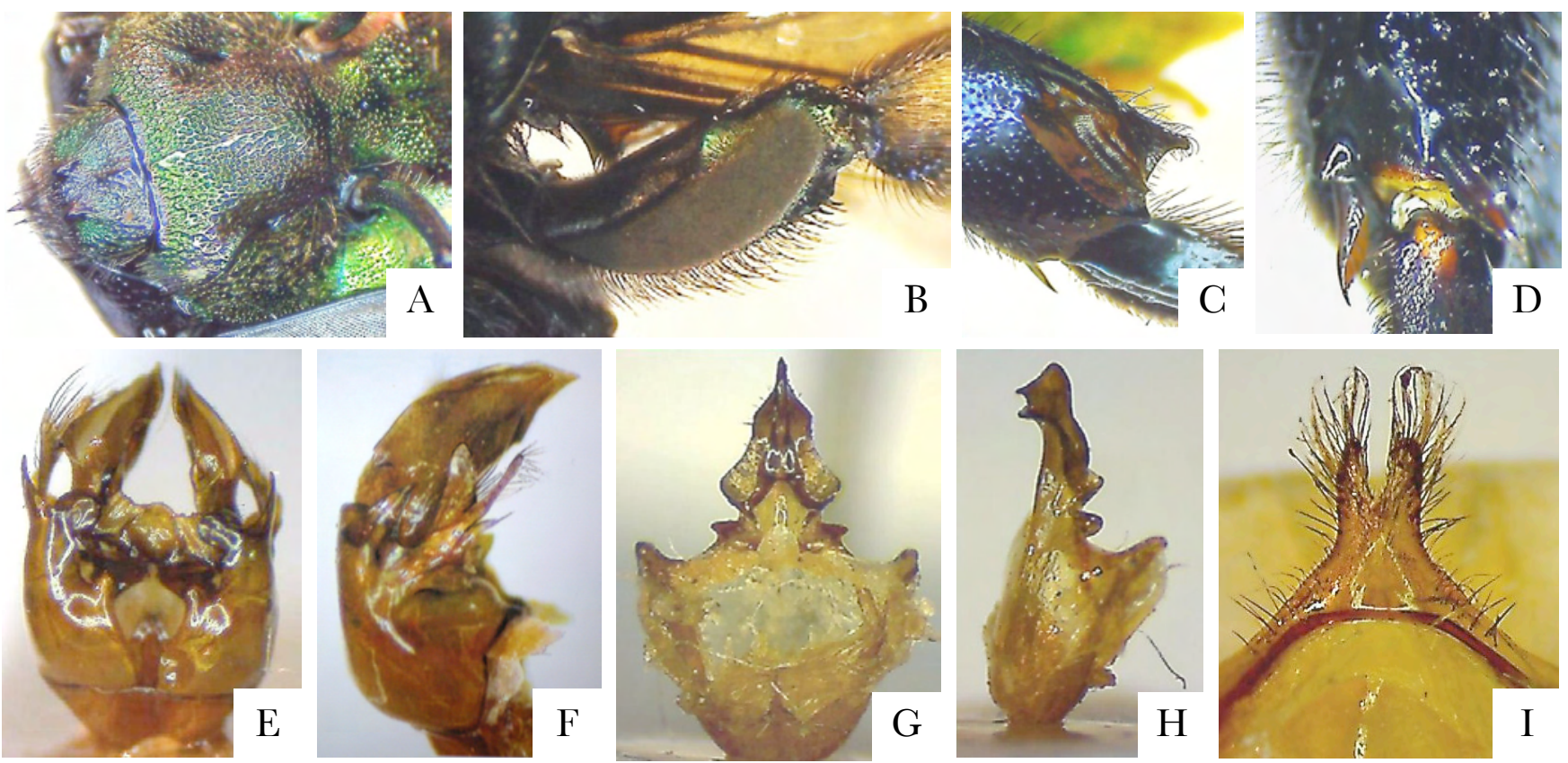

Figure 2. Détails morphologiques diagnostiques de l'allotype mâle d'Eufriesea formosa MocsARY. A. Labrum et clypeus; B. Zones veloutées du fémur médian et brosse basitibiale à droite; C. Patte postérieure - extrémité du fémur en vue dorsale; D. Patte postérieure - extrémité du fémur en vue ventrale; E. Genitalia en vue dorsale; F. Genitalia en vue de profil; G. Sternite 8 en vue ventrale; H. Sternite 8 en vue de profil; I. Sternite 8 en vue ventrale (Photos G Le Goff). 